\title{
PERIOPERATIVE PROPHYLACTIC CHEMOTHERAPY OF ECHINOCOCCUS GRANULOSUS: DETERMINATION OF MINIMUM EFFECTIVE LENGTH OF ALBENDAZOLE THERAPY IN IN VITRO PROTOSCOLEX CULTURE
}

\author{
D.H. TAYLOR ${ }^{1}$, D.L. MORRIS ${ }^{1}$ and K.S. RICHARDS ${ }^{2}$ \\ ${ }^{1}$ Department of Surgery, University Hospital, Nottingham NG7 2UH, UK. \\ ${ }^{2}$ Department of Biological Sciences, University of Keele, Keele, Staffs. ST5 5BG, UK.
}

(Received 15 July 1989)

\begin{abstract}
Protoscoleces of Echinococcus granulosus were cultured in vitro in 500,250 or $100 \mu \mathrm{g} / 1$ albendazole sulphoxide for $1,3,7,10,14 \mathrm{~d}$ and then 'rescued' $(R)$ into drug-free medium for the remainder of the culture period. Successful minimum lengths of therapy were much longer than for praziquantel, and only at $500 \mu \mathrm{g} / 1$ was the $10 \mathrm{dR}$ treatment as effective as continuous therapy for $28 \mathrm{~d}$. Treatment with $100 \mu \mathrm{g} / \mathrm{l}$ both in continuous culture and in the ' $R$ ' experiments was ineffective over a $35 \mathrm{~d}$ period. The results are compared with those from similar experiments using praziquantel.
\end{abstract}

KEY WORDS: Albendazole, praziquantel, protoscoleces

\section{INTRODUCTION}

Chemotherapy against hydatid disease in man is not without risk, and it is clearly important to know the minimum length of time required for effective therapy. This paper studies the duration of exposure to albendazole needed to kill protoscoleces, as would be necessary in a prophylactic course in patients following operation/spillage. We have previously reported that albendazole sulphoxide, the active metabolite of albendazole, is an effective in vitro protoscolicidal agent. Approximately $250 \mu \mathrm{g} / \mathrm{l}$ albendazole sulphoxide is required to produce a significant reduction in viability after 31 days $^{1,2}$. We have also found praziquantel to be a most active protoscolicidal agent and have demonstrated that in in vitro the effect of comparatively short-term exposure to the drug causes irreversible damage, and that protoscoleces so treated and then 'rescued' into drug-free media continue to die $^{3}$. It was therefore decided to study short lengths of exposure to various concentrations of albendazole sulphoxide followed by the 'rescue' of the protoscoleces into drug-free medium to establish the minimum exposure time necessary for effective treatment by albendazole sulphoxide.

\section{MATERIALS AND METHODS}

Protoscoleces were collected from both hepatic and pulmonary hydatid cysts in naturally infected sheep obtained from a local abattoir and maintained at $37^{\circ} \mathrm{C}$ in NCTC135 (Gibco) with $20 \%$ heat-inactivated foetal calf serum (Gibco) as previously 
described ${ }^{4}$. Only cultures with an initial viability of over $95 \%$ as judged by microscopy/ eosin exclusion were used. Albendazole sulphoxide (Smith, Kline \& French) concentrations of 500,250 and $100 \mu \mathrm{g} / \mathrm{l}$ were studied together with controls to which an identical volume of solvent ( $50 \%$ methanol) had been added. The final concentration of methanol in the culture medium of both test and control tubes was $2.5 \mathrm{ml} / 1$ and this has no significant effect on protoscolex viability ${ }^{5}$. A minimum of 10 replicates at each drug concentration was performed. All media were changed and protoscolex viability assessed by microscopy/eosin exclusion every 3-4 days.

At days 1, 3, 7, 10 and 14 individual tubes of treated protoscoleces at each concentration were 'rescued' into drug-free medium for the remainder of the experimental period ( 28 days for $500 \mu \mathrm{g} / \mathrm{l}, 35$ days for 250 and $100 \mu \mathrm{g} / \mathrm{l})$. These will be referred to as $1 \mathrm{dR}$ ( 1 day Rescue), $3 \mathrm{dR}, 7 \mathrm{dR}$ etc. respectively in contrast to tubes receiving continuous drug therapy.

Statistical analysis of viability data for control and treated cultures was done using a GLIM statistical package (GLIM 3.77 update), 1985, Royal Statistical Society, London) to fit logistic regression lines with the proportion of alive protoscoleces as the dependent variable and the number of days in in vitro culture as the independent variable, with treatments groups as a factor to allow cross treatment comparisons.

\section{RESULTS}

Figures 1-3 present the viability of protoscoleces treated with 500,250 and $100 \mu \mathrm{g} / 1$ albendazole sulphoxide and rescued into drug-free medium at days $1,3,7,10$ and 14 together with control and continuous therapy cultures.

At a concentration of $500 \mu \mathrm{g} / 1$ albendazole sulphoxide, neither 1 nor 3 days culture with the drug (1dR and $3 \mathrm{dR}$ ) before rescue caused any significant reduction in protoscolex viability over the 28 day culture period. The $7 \mathrm{dR}$ results showed a strong trend towards being different from the controls but were not significantly different ( $p$ $<0.1$ ). The $10 \mathrm{dR}$ treatment not only significantly reduced protoscolex viability when compared with the controls $(\mathrm{p}<0.01)$ but was also significantly better than the $7 \mathrm{dR}$ therapy $(p<0.05)$. Likewise, $14 \mathrm{dR}$ was significantly more effective than both controls and $10 \mathrm{dR}(\mathrm{p}<0.01)$. Continuous therapy was more effective than controls, $1 \mathrm{dR}, 3 \mathrm{dR}$ and $7 \mathrm{dR}(\mathrm{p}<0.01)$, but not significantly more than $10 \mathrm{dR}$ and $14 \mathrm{dR}(\mathrm{p}<0.1)$, though a strong trend was apparent.

At a concentration of $250 \mu \mathrm{g} / \mathrm{l}$ of albendazole sulphoxide, only $1 \mathrm{dR}$ was not significantly different from the control (3dR $\mathrm{p}>0.025 ; 7 \mathrm{dR}, 10 \mathrm{dR}$ and $14 \mathrm{dR} \mathrm{p}<$ 0.001 ), and each increase in duration of drug treatment achieved a significantly greater reduction in protoscolex viability.

The apparently greater effect of albendazole $250 \mu \mathrm{g} / 1$ compared with $500 \mu \mathrm{g} / \mathrm{l}$ was due to a larger SD in the $500 \mu \mathrm{g} / \mathrm{l}$ controls and if the horizontal 'end viability' portion of the percentage viability curves are studied it will be seen that whilst Alb Sx $500 \mu \mathrm{g} / \mathrm{l}$ achieved viability of $20 \%$ or less after $7,10,14$ or continuous Alb Sx $\mu \mathrm{g} / 17 \mathrm{dR}$ achieved approximately $40 \%, 10 \mathrm{dR}$ approximately $30 \%$ and it was only $14 \mathrm{dR}$ and continuous therapy that achieved $20 \%$ or less.

At a concentration of $100 \mu \mathrm{g} / \mathrm{l}$ of albendazole sulphoxide there was no significant difference between any of the experimental groups and the control cultures, even at the end of the 35 day culture period. 


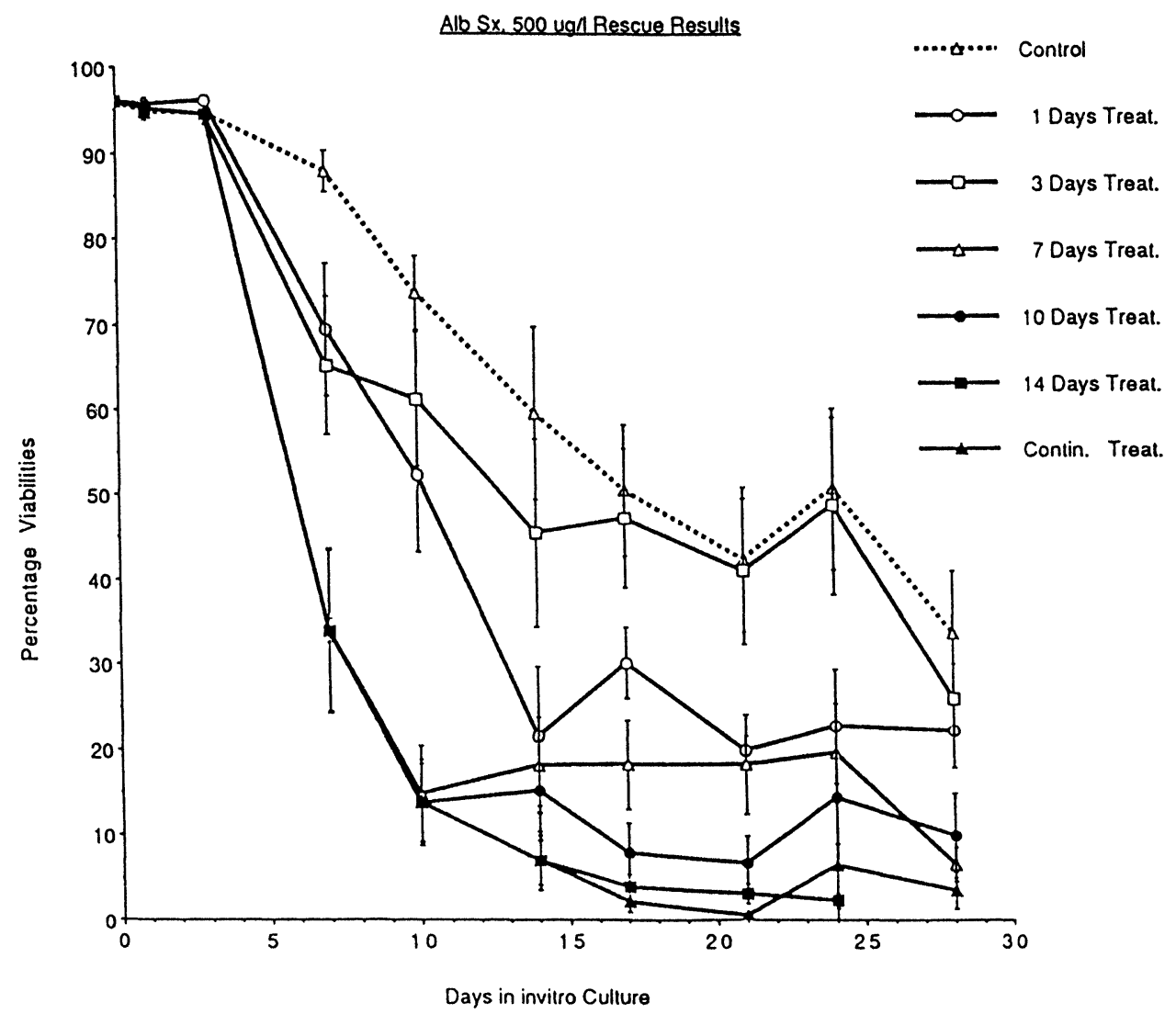

Figure 1. Effect of $500 \mu \mathrm{g} / 1$ albendazole sulphoxide for $28 \mathrm{~d}$, and for $1,3,7,10,14 \mathrm{~d}$ followed by drug-free culture on the viability of protoscoleces of E. granulosus.

\section{DISCUSSION}

It has already been established that albendazole sulphoxide is protoscolicidal when used in vitro over a 30 day period ${ }^{1}$. It was not clear, however, whether this length of treatment was necessary for a drug-induced injury to cause death of the protoscoleces or whether continuous treatment was actually necessary to achieve the lethal effect.

In vitro culture of protoscoleces of $E$. granulosus has allowed us to study various drug concentrations and lengths of therapy. It must be accepted however that each experiment is different and that because of batch to batch variations in both the viability and survival of protoscoleces, controls must be maintained in each experiment and reduction in viability of treated cultures compared with the viability of control cultures within the same experiment.

The present study has shown that continued exposure to albendazole sulphoxide is required, and that after drug withdrawal there is little if any further decrease in 


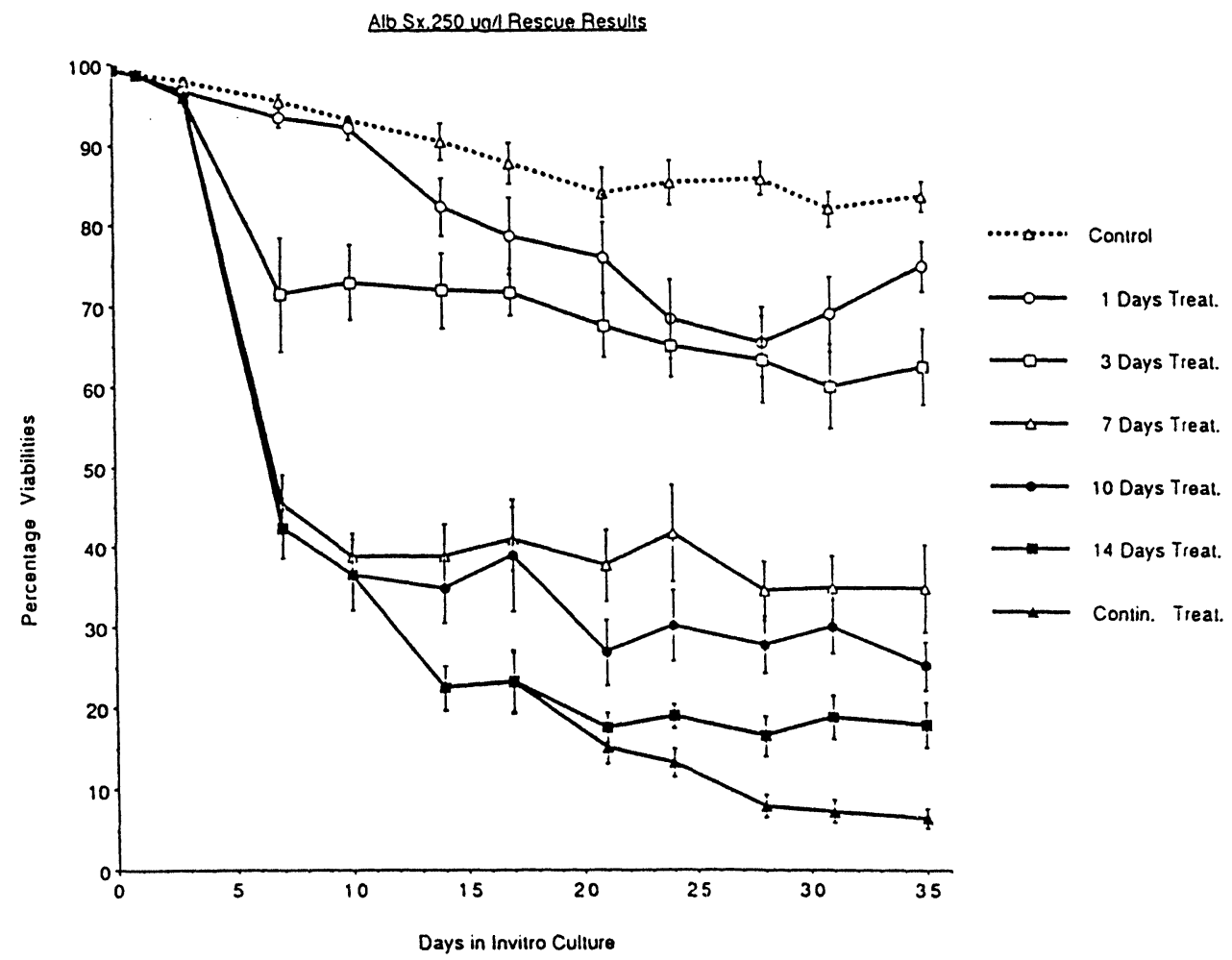

Figure 2. Effect of $250 \mu \mathrm{g} / \mathrm{l}$ albendazole sulphoxide for $35 \mathrm{~d}$, and for $1,3,7,10,14 \mathrm{~d}$ followed by drug-free culture on the viability of protoscoleces of $E$. granulosus.

protoscolex viability. This is different from praziquantel where very short lengths of treatment are lethal ${ }^{3}$ and exposure times of as short as $10 \mathrm{~min}$ are effective at very high concentrations ${ }^{6}$. This difference between albendazole, a benzimidazole carbamate, and praziquantel, an isoquinoline, is probably related to the different mechanism of action of these compounds. Praziquantel is known to have a disruptive effect on the tegument of both the adult and protoscolex stage of $E$. granulosus ${ }^{7-10}$ whereas benzimidazoles are thought to bind to tubulin ${ }^{11}$ and inhibit its polymerization, and thus have a more gradual effect on parasite tissues. The results of the current 'rescue' type experiments would seem to support this in that prolonged therapy with albedazole sulphoxide seems to be necessary in order to achieve the same degree of protoscolicidal efficacy as that brought about by much shorter periods of treatment with praziquantel. The administration of albendazole as an intraoperative agent is therefore unlikely to be effective, but its possible role in postoperative therapy might still be considered. 


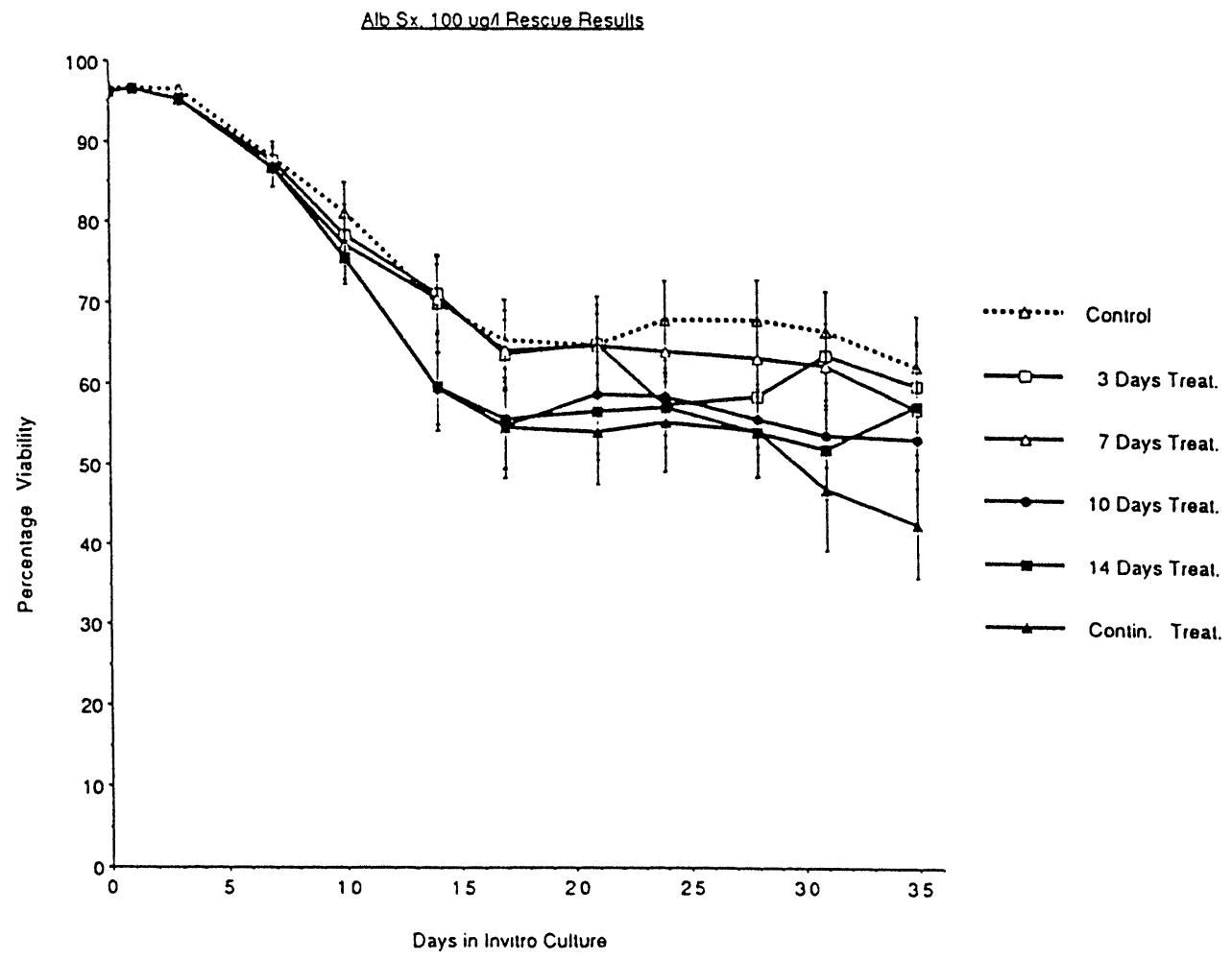

Figure 3. Effect of $100 \mu \mathrm{g} / 1$ albendazole sulphoxide for $35 \mathrm{~d}$, and for $1,3,7,10,14 \mathrm{~d}$ followed by drug-free culture on the viability of protoscoleces of E. granulosus.

\section{Acknowledgements}

Smith, Kline \& French are thanked for their financial support and for the supply of albendazole sulphoxide.

\section{References}

1 Chinnery,J.B. andMorris,D.L.,Effect of albendazolesulphoxide onviability of hydatidprotoscolecesin vitro, Trans. Roy. Soc. Trop. Med. Hyg., 1986; 80: 815-817.

2 Morris, D.L., Chinnery, J.B. and Ubhi, C., A comparison of the effects of albendazole, its sulphone metabolite and mebendazole on viability of Echinococcus granulosus protoscoleces in an in vitro culture system, Trans. Roy. Soc. Trop. Med. Hyg.,, 1987; 81: 804-806.

3 Morris, D.L., Taylor, D.H., Daniels, D., et al., Determination of the minimum time of praziquantel therapy required for the in vitro treatment of protoscoleces of Echinococcus granulosus, J. Helminth., 1988; 62: 10-14.

4 Taylor, D.H., Morris, D.L. and Richards, K.S., Combination chemotherapy of Echinococcus granulosus - in vitro studies, Trans. Roy. Soc. Trop. Med. Hyg., 1988 82: 263-264. 
5 Taylor, D.H. and Morris, D.L., In vitro culture of Echinococcus multilocularis: protoscolicidal action of praziquantel and albendazole sulphoxide, Trans. Roy. Soc. Trop. Med. Hyg., 1988; 82: 265-267.

6 Richards, K.S., Riley, E.M., Taylor, D.H. and Morris, D.L., Studies on the effect of short term, high dose praziquantel treatment against protoscoleces of ovine and equine Echinococcus granulosus within the cyst, and in vitro, Trop. Med. Parasit., 1988; 39: 269-272.

7 Becker, B., Mehlhorn, H., Andrews, P. and Thomas, H., Ultrastructural investigations on the effect of praziquantel on the tegument of five species of cestodes, $Z$. Parasitenkd, 1981; 64: 257-269.

8 Conder, G.A., Marchiondo, A.A. and Andersen, F.L., Effect of praziquantel on adult Echinococcus granulosus in vitro: scanning electron microscopy, Z. Parasitenkd, 1981; 66: 191-199.

9 Morris, D.L., Richards, K.S. and Chinnery, J.B., Protoscolicidal effect of praziquantel - in vitro and electron microscope studies on Echinococcus granulosus, J. Antimicr. Chem., 1986; 18: 687-691.

10 Morris, D.L., Taylor, D.H., Daniels, D. and Richards, K.S., Determination of minimum effective concentrations of praziquantel in in vitro cultures of protoscoleces of Echinococcus granulosus, Trans. Roy. Soc. Trop. Med. Hyg.,., 1987; 81: 494-497.

11 Laclette, J.P., Guerra, G. and Zetina, C., Inhibition of tubulin polymerization by mebendazole, Biochem. Biophys. Res. Comm., 1980; 92: 417-423.

(Accepted by S. Bengmark 27 July 1989) 


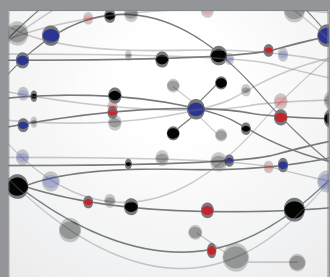

The Scientific World Journal
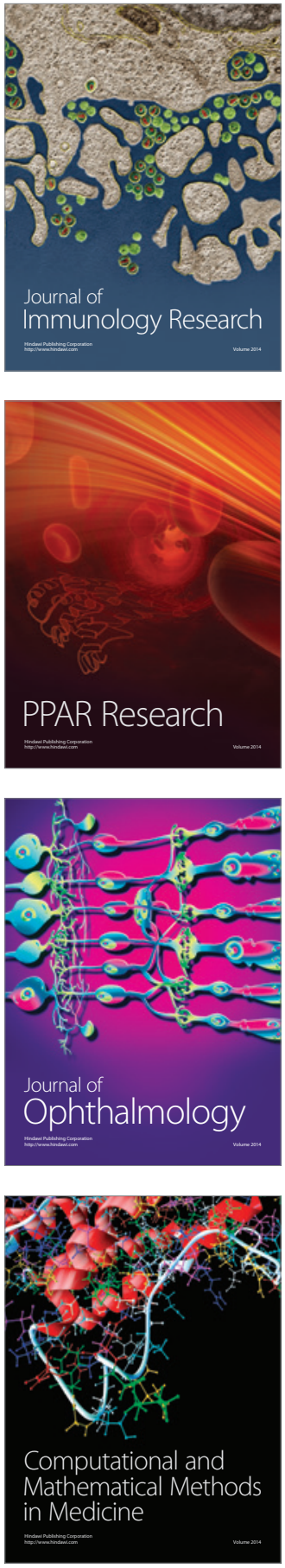

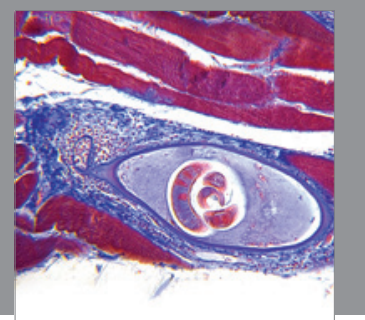

Gastroenterology

Research and Practice
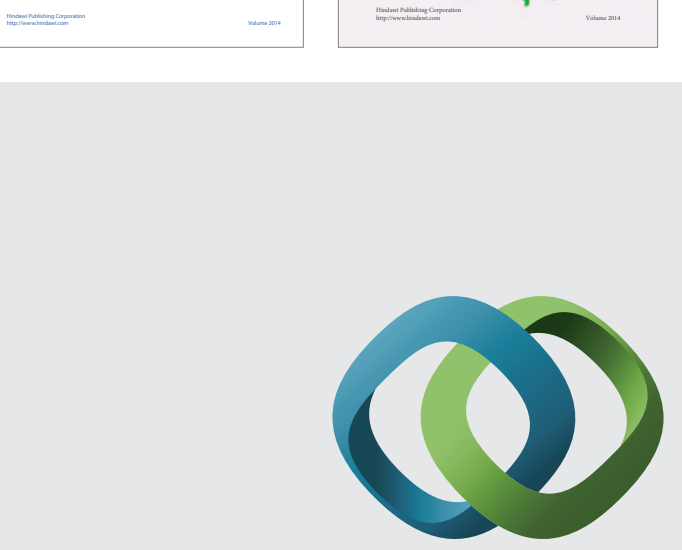

\section{Hindawi}

Submit your manuscripts at

http://www.hindawi.com
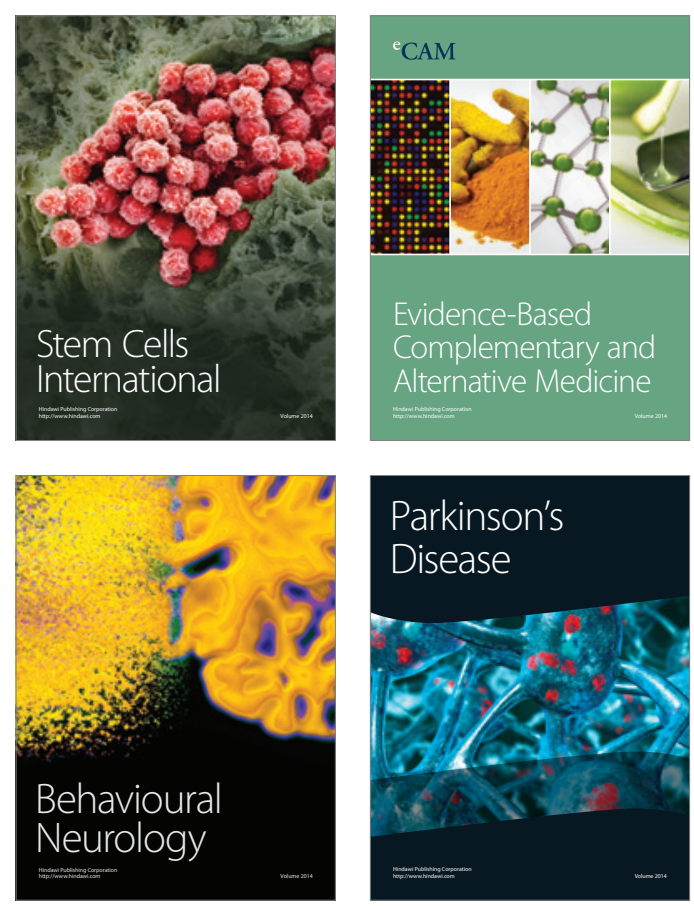

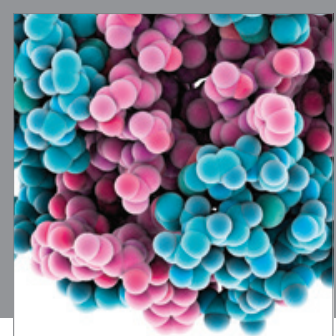

Journal of
Diabetes Research

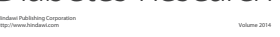

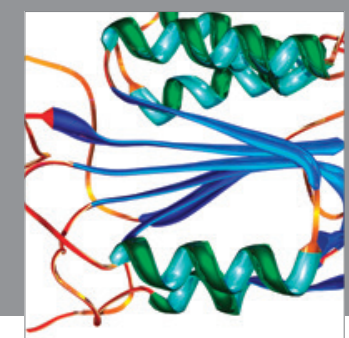

Disease Markers
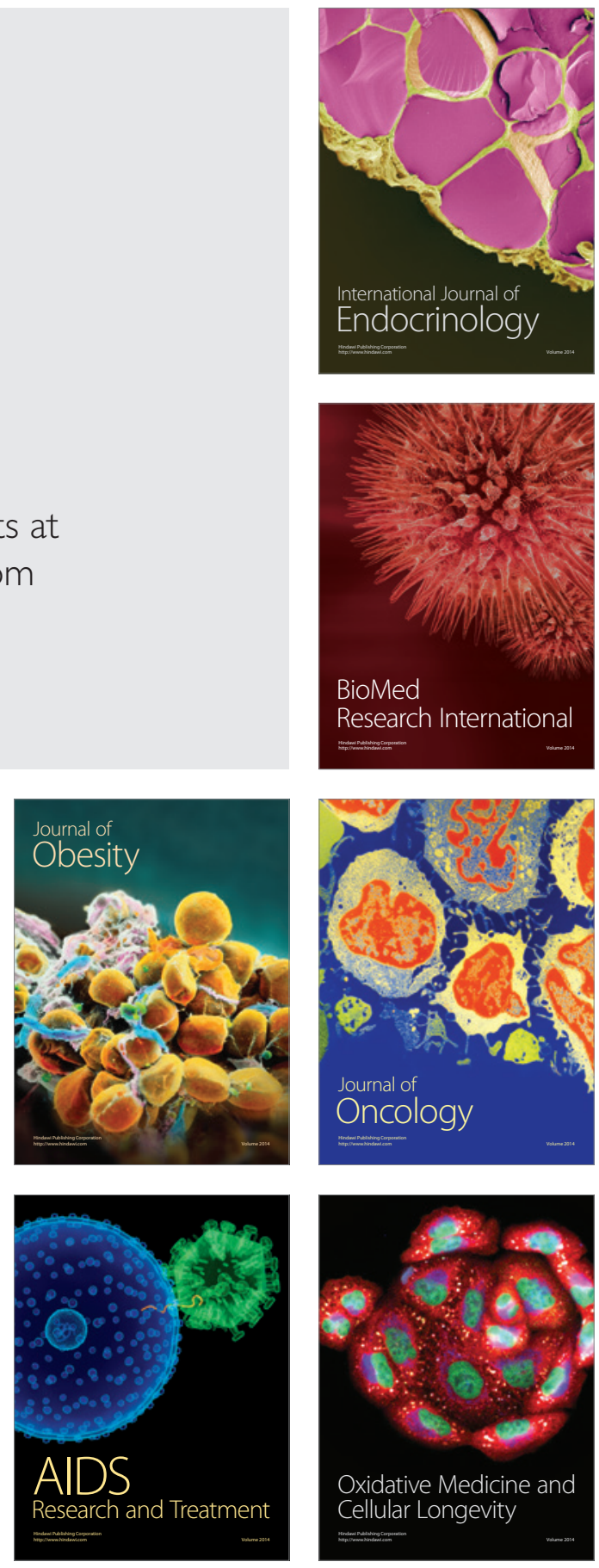